\title{
ANÁLISIS EPISTEMOLÓGICO DEL CURRICULUM DE FORMACIÓN DOCENTE: EL CASO DE LA SOCIOLOGÍA DE LA EDUCACIÓN EPISTEMOLOGICAL STUDY OF TEACHER EDUCATION CURRICULUM: THE EXAMPLE OF EDUCATIONAL SOCIOLOGY
}

\author{
Volumen 14, Número 2 \\ Mayo - Agosto \\ pp. 1-20
}

Este número se publicó el 30 de mayo de 2014

María Gisella Boarini ${ }^{1}$

Revista indizada en REDALYC, $\underline{\text { SCIELO }}$

Revista distribuida en las bases de datos:

CATÁLOGO DE LATINDEX, IRESIE, CLASE, DIALNET, DOAJ, E-REVIST@S, SHERPA/ROMEO, QUALIS, MIAR

Revista registrada en los directorios:

ULRICH'S, REDIE, RINACE, OEI, MAESTROTECA, PREAL, CLASCO 


\section{ANÁLISIS EPISTEMOLÓGICO DEL CURRICULUM DE FORMACIÓN DOCENTE: EL CASO DE LA SOCIOLOGÍA DE LA EDUCACIÓN \\ EPISTEMOLOGICAL STUDY OF TEACHER EDUCATION CURRICULUM: THE EXAMPLE OF EDUCATIONAL SOCIOLOGY}

\section{María Gisella Boarini ${ }^{1}$}

Resumen: El presente ensayo procura develar algunas cuestiones epistemológicas que subyacen a los estudios educativos y que tienen consecuencias en la práctica y en la formación de docentes. Se parte del caso de la Sociología de la Educación para mostrar la inconmensurabilidad y la falta de unidad que en muchas ocasiones atraviesan a algunas ciencias de la educación. Para ello, se realiza un estudio del origen y el desarrollo histórico de la Sociología de la Educación como disciplina científica. Este análisis permite comprender el surgimiento y los alcances de los problemas epistemológicos mencionados, principalmente en la formación docente.

Palabras clave: CIENCIAS DE LA EDUCACIÓN, SOCIOLOGÍA EDUCATIVA, PEDAGOGÍA, FORMACIÓN DOCENTE, EPISTEMOLOGÍA EDUCATIVA

Abstract: The purpose of this essay is to show some epistemological issues which underlie educational studies which have consequences in teacher training. Based on Sociology of Education, it shows the incommensurability and lack of unity that usually affect some Sciences of Education. A study of the origin and historic development of Educational Sociology as a science is carried out. This analysis enables us to understand the origin and significance of the epistemological problems mentioned before, especially in teacher training.

Key words: SCIENCES OF EDUCATION, EDUCATIONAL SOCIOLOGY, PEDAGOGY, TEACHER TRAINING, EDUCATIONAL EPISTEMOLOGY

\footnotetext{
1 Investigadora del Instituto de Investigación de Ciencias de la Educación de la Universidad Nacional de Cuyo (Argentina). Licenciada en Ciencias de la Educación. Becaria del Consejo Nacional de Investigaciones Científicas y Técnicas (CONICET).
}

Dirección electrónica: mgboarini@gmail.com

Ensayo recibido: 9 de abril, 2013

Devuelto para corrección: 9 de julio, 2014

Aprobado: 15 de mayo, 2014 


\section{Introducción}

El examen de los fundamentos epistemológicos que subyacen a la investigación y a la práctica educativa posibilita la comprensión de los elementos que le dan una forma determinada al campo educativo. En el presente ensayo se realizará un análisis del origen y desarrollo de la Sociología de la Educación como disciplina científica, desde principios del siglo XX hasta la actualidad. Este estudio permitirá sacar a la luz algunas consecuencias epistemológicas que contribuyen a comprender la configuración actual de esta disciplina dentro del conjunto de las Ciencias de la Educación y que, por lo tanto, enriquecen la investigación dentro de ella.

\section{Los orígenes de la Sociología de la Educación como campo disciplinar}

\subsection{Comienzo positivista}

A principios del siglo XX Durkheim inaugura en Francia una nueva discusión al introducir el término de ciencia de la educación y oponerlo al de pedagogía, concepto que se venía utilizando hasta el momento. En efecto, la Pedagogía como ciencia había nacido, en la tradición alemana iniciada por Herbart, ligada a la filosofía, más precisamente a la ética, y a la psicología. De este modo, abordaba la educación desde concepciones sobre el hombre y su deber ser para intentar normar el acto educativo.

Esta nueva ciencia de la educación propuesta por Durkheim debía transformarse en una ciencia positiva, basada en hechos, que permitiera describir de modo causal la realidad educativa como hecho social. Esto implicaría estudiar los fenómenos humanos y sociales al modo de las ciencias de la naturaleza, es decir, describiendo sus causas físicas y observables, en contraposición a las ciencias humanas que buscan una comprensión histórico-hermenéutica.

Se observa claramente la relación directa entre la sociología comenzada por Comte y la ciencia de la educación propuesta por Durkheim. En efecto, este último fundamenta intencionalmente la ciencia de la educación en la Sociología y, por lo tanto, en la tradición positivista. Por ello, suele considerarse a Durkheim como el iniciador de la Sociología de la Educación, a pesar de no haberla denominado él de esa manera. Al respecto afirma Lerena:

No se trata de que Saint-Simón o Comte además de fundar la sociología y de ocuparse de tal o cual aspecto de la realidad se ocupasen también de la educación [...] Se trata de que el planteamiento epistemológico que dio origen a lo que llamamos sociología contenía ya en su raíz una [...] determinada concepción de la educación y de la 
escuela, y esa determinada concepción constituye el núcleo en torno al cual elaboran su posterior reflexión [...] Durkheim, Mannheim o Parsons. (en Guerrero Serón, 1996, p. 63)

En esta nueva ciencia de la educación, que posteriormente comenzará a llamarse Sociología de la Educación, cambia el centro de los planteos: ya no es la formación del hombre (finalidad de la Pedagogía alemana) lo que se estudia, sino la educación en una sociedad determinada como hecho o fenómeno; por eso ya no se necesita a la Filosofía como ciencia de base, sino a la Sociología².

Hay dos hitos fundamentales en los orígenes de la nueva disciplina: en 1902 Durkheim pronuncia un discurso sobre Pedagogía y Sociología al asumir como profesor auxiliar de la cátedra de Ciencia de la Educación ${ }^{3}$ en la Sorbona (aunque ya había dictado algunos cursos de Pedagogía en la Universidad de Burdeos desde 1887). Por su lado, en 1922 se publica póstumamente su libro Educación y Sociología, en el que establece el objeto de lo que se llamará Sociología de la Educación y la distingue de la Pedagogía.

Es fundamental profundizar en el planteo de Durkheim, ya que la orientación que él dio a la ciencia de la educación es uno de los fundamentos de la actual conformación de las Ciencias de la Educación como conjunto.

En cuanto a su concepción antropológica, sostiene que en el hombre coexisten dos seres (distintos, pero solo separables por abstracción). Uno es el ser individual, innato, constituido por las cualidades físicas y mentales que se refieren únicamente al hombre mismo. El ser social, en cambio, es adquirido y está formado por el conjunto de ideas, costumbres, sentimientos y hábitos que expresan el grupo o los grupos de los que el sujeto forma parte. Por esta razón, el hombre es naturalmente un ser individual, asocial y egoísta y es la sociedad la que forma en él, por medio de la educación, un cierto ideal de hombre, "de lo que éste debe ser, tanto desde el punto de vista intelectual como físico y moral" (Durkheim, 1976, p. 68).

Por lo tanto, si es la sociedad la que determina qué debe ser el hombre y, en consecuencia, cómo debe ser educado, está claro que su postura pedagógica se apoya, no

\footnotetext{
${ }^{2}$ Específicamente, la filosofía que se descarta es aquella que, por su naturaleza metafísica, es considerada falsa. Cabe recordar que Comte acepta como verdadera filosofía a la filosofía positiva y funda la sociología considerándola como la más importante rama de esta filosofía. Por eso, el enfoque positivo dado a la ciencia de la educación es el único que puede ser verdadero.

${ }^{3}$ Algunos autores afirman que la denominación de esta cátedra es Sociología. Sin embargo, Paul Fauconnet dice, en la introducción que realiza al libro de Durkheim Educación y Sociología, que éste "en la Sorbona fue auxiliar de la cátedra de Ciencia de la Educación en 1902".
} 
solo en su modo de concebir al hombre, sino también en su teoría sobre la sociedad. Esta es entendida como un todo que se consolida por la solidaridad, la cooperación entre las personas. En una sociedad atrasada esta solidaridad es mecánica, basada en la semejanza y la cohesión producida por una conciencia religiosa común. La complejización de las sociedades y su crecimiento demográfico produce una especialización y división del trabajo social. En este caso predomina la solidaridad orgánica, fundada en la interdependencia de los sujetos y de sus tareas especializadas. Esta interdependencia es necesaria para vivir; sin embargo, provoca un deterioro de los lazos entre los individuos y una creciente desigualdad, lo que culmina muchas veces en situaciones de anomia o ausencia de normas de referencia y pautas de comportamiento.

Aquí es donde hace su entrada la educación, como parte de la solución a estos problemas. Por un lado, forma a los individuos en la especialidad de los distintos trabajos necesarios para la interdependencia funcional dentro de las nuevas sociedades complejas; por otro, transmite la cultura y la moral colectiva que lleven a la cohesión social.

Por consiguiente, la educación no es natural sino social, es decir, se crea a través de ella "el ser social que hay en cada persona" (Guerrero Serón, 1996, p. 63). En conclusión, la educación es la acción ejercida por las generaciones adultas sobre "aquellos que no han alcanzado todavía el grado de madurez necesaria para la vida social. Tiene como objeto suscitar y desarrollar en el niño un cierto número de estados físicos, intelectuales y morales que exigen de él, tanto la sociedad política en su conjunto, como el medio ambiente específico al que especialmente está sometido" (Durkheim, 1976, p. 70).

Si la educación es un hecho exclusivamente social, dependerá totalmente de la sociedad concreta y del momento histórico-cultural en el que se desarrolle. De ahí que el fin de la educación no puede ser "el desarrollo armónico de todas las facultades [del hombre] [...] Este desarrollo armónico es, en efecto, necesario y deseable, pero no es integralmente realizable" (Durkheim, 1976, p. 56-57). Este es un punto crucial de distanciamiento entre Durkheim y la tradición alemana de pedagogía. En efecto, en esta se buscaba como finalidad última de todo proceso educativo la formación del carácter moral 0 , en palabras de Durkheim, el desarrollo armónico de todas las facultades del hombre. Este fin era válido para todo hombre, para un hombre universal. Por lo tanto, para alcanzarlo, la pedagogía debía valerse de la filosofía, más precisamente de la ética. Por el contrario, en la incipiente ciencia de la educación francesa, el fin de la educación es la socialización, esto es, un proceso mediante el cual se forma en el sujeto el "ser social". Este hace referencia al modelo que la 
sociedad, por medio de la conciencia colectiva, adopta como valor e impone al sujeto. Este fin no alcanza a todo hombre en todo tiempo, sino que depende necesariamente de la sociedad concreta y del momento histórico en los que el individuo viva.

Como la educación es socialización, es decir, transmisión de los valores de una sociedad, es principalmente educación moral. La moral "cambia cuando las sociedades cambian" (Durkheim, 1976, p. 78), por lo que los fines, medios y valores educativos son determinados por las circunstancias histórico-sociales.

Ahora bien, después de todo lo dicho se hace impensable para Durkheim sostener una Pedagogía normativa que se ocupe de lo que debe ser y no de lo que es (hechos sociales). Por esta razón, plantea la necesidad de contar con una ciencia de la educación que cumpla con los requisitos de toda ciencia positiva, a saber: que verse sobre hechos observables, que estos hechos presenten entre sí la suficiente homogeneidad para que se les pueda clasificar en una misma categoría y que la finalidad ulterior sea simplemente conocer de manera desinteresada.

En su intento de fundamentar científicamente la educación, Durkheim propone dos ciencias de apoyo. Una es la psicología, que es útil para proveer los medios y procedimientos adecuados de educación. La otra es la sociología, de quien depende la pedagogía, según este autor, más estrechamente que de cualquier otra ciencia, ya que puede determinar el fin y la orientación general de los métodos educativos.

Después de haber quedado así establecida la ciencia de la educación, el pasaje a ciencias de la educación se realiza efectivamente en 1967 cuando Debesse crea una maestría con ese nombre. El plural "parece responder a una apertura del campo a los aportes no solo de la sociología, sino de la psicología, la biología y ya posteriormente la antropología cultural, la lingüística, etc." (Vázquez, 2001, p. 15). Este plural manifiesta la diferenciación con el término pedagogía y con el tinte normativo y filosófico del mismo. El objeto propio de las ciencias de la educación es el "estudio del funcionamiento del fenómeno educativo y de las instituciones a que da lugar en los diversos contextos socio-culturales, con una metodología científica (es decir causal y no simplemente descriptiva)." (Vázquez, 2001, p. 13).

\subsection{El optimismo norteamericano}

A principios del siglo XX aparece en los Estados Unidos, paralelamente a los estudios sociológicos de Francia, la corriente conocida como Educational Sociology (Sociología 
Educacional o Pedagógica), cuya preponderancia va a extenderse hasta la década del sesenta. A fines del siglo XIX se dictan una serie de cursos y cátedras relativos a esta nueva disciplina y en 1927 Payne funda la primera revista destinada a esta temática: Journal of Educational Sociology. En esta línea, la sociología se presenta como una "ciencia de apoyo para la educación" (Guerrero Serón, 1996, p. 63), pero siempre ligada a estudios pedagógicos. Su rasgo sobresaliente es su carácter descriptivo y pragmático: se realiza un estudio de la educación norteamericana con el objeto de reformarla; es una sociología aplicada a la educación o, mejor dicho, una pedagogía que realiza investigaciones sociales. Este pragmatismo responde a un progresismo liberal que encuentra la solución a los problemas sociales en la escuela, en tanto factor de socialización. Algunos autores más contemporáneos han seguido esta vertiente denominándola Pedagogía Social y considerándola una pedagogía con apoyo sociológico y con una intencionalidad normativa.

Esta corriente ha sido caracterizada como de orientación pedagógica. En los años de la posguerra, debido a la complejización de la estructura social propiciada por la industrialización y al desarrollo de los sistemas económicos y educativos nacionales, renace la Sociología de la Educación para estudiar el progreso de la institución escolar, sus resultados y su relación con la organización económica y social. En este sentido, afirma Rodríguez Sedano (2006, p. 132) que "el origen de la Pedagogía Social está vinculado a la necesidad de intervenir socioeducativamente en una sociedad en crisis, como consecuencia de las profundas alteraciones producidas por la industrialización [...] El auge de la Pedagogía Social en el siglo veinte se vincula con el desarrollo tecnológico y su actual expansión en el proceso de globalización".

En este auge descrito aparecen sociólogos que consolidan la disciplina y le imprimen una orientación sociológica, tales como Parsons (EEUU), Fischer y Mannheim (Alemania). Este nuevo carácter es visto por los autores que actualmente se dedican a la disciplina como el auténtico, ya que la finalidad dejó de ser normar para alcanzar ciertos fines educativos, sino explicar, describir y comprender el hecho educativo en tanto hecho social. El cambio mencionado "puede señalarse oficialmente en el año 1963, en que el Journal of Educational Sociology cambia su nombre por el de Sociology of Education." (Quintana Cabanas, 1989, p.45).

En este marco, cabe añadir los estudios realizados en la década del cincuenta en los países anglófonos sobre la política económica de acceso a la educación. La corriente a la que se hace referencia es el funcionalismo, que se interesa por considerar las funciones 
sociales de la educación en el contexto específico de la sociedad americana. En los años posteriores a la Segunda Guerra Mundial se vivió en Norteamérica una transformación del papel social de la educación, que se observó tanto en el crecimiento de la demanda como de la oferta de educación y en el aumento de inversión pública y privada en materia educativa. Esta gran expansión de la cobertura educativa se debió a varios factores: por un lado, la educación mejoraba la calidad del trabajo y, por lo tanto, la productividad del mismo; por otro, la educación formal fue presentada como el principal factor de movilidad social. Esto, gracias a su doble función de socialización y diferenciación. La primera se realiza al transmitir los valores de la sociedad y las habilidades para el ejercicio profesional futuro; la diferenciación y selección social se llevan a cabo a través de la distribución de premios y castigos que conduce a la asignación de diferentes posiciones dentro de la estructura social. En función de lo dicho, el saber sociológico aplicado a la educación se reconoció como el interés principal del funcionalismo: ya no era el saber pedagógico, sino el sociológico el que servía como base de legitimación científica de las decisiones políticas.

Una idea central en la perspectiva funcionalista es la desarrollada por Parsons: la meritocracia. Este concepto indica que las posiciones sociales se corresponden con la capacidad y el esfuerzo personal, no según un principio hereditario. Ahora bien, el medio para adquirir y desarrollar estas capacidades es la educación formal. De ahí que la igualdad de oportunidades sea una condición indispensable que debe ser garantizada por el Estado.

Esta posición tuvo varias expresiones, por ejemplo, la teoría del capital humano de Gary Becker, según la cual la mejor inversión que se puede hacer es la que se realiza en la educación y formación del factor humano de la producción. En Gran Bretaña, Mannhein (exiliado de Alemania), Floud y Halsey atienden a la igualdad de oportunidades, la democratización de la enseñanza y las relaciones entre educación y movilidad social. Posteriormente, Coleman y Jencks (EEUU) estudian las relaciones entre desigualdad educativa y social.

Años más tarde Collins (1970-1990) habla de Credencialismo. Esta perspectiva sostiene que el otorgamiento de credenciales (títulos, diplomas) por parte de la escuela es el factor de selección y clasificación social. Se afirma aquí que la estratificación social se ha debido más a una pugna por mantener las clases dominantes su posición que a una selección según las capacidades personales y los requerimientos técnicos y económicos de la sociedad. 
Los títulos académicos constituyen, ante todo, las credenciales que prueban un determinado estilo cultural y los requisitos formales más importantes del empleo. [...] los empresarios valoran los títulos, pero no como indicadores de la competencia profesional de cada uno, sino como garantía del dominio personal de un determinado estilo de vida. Puesto que los requisitos cognitivos específicos de la mayor parte de los empleos continúan aprendiéndose fundamentalmente en el puesto de trabajo, [...] los títulos -al menos los más codiciados de ellos- constituirían sobre todo una garantía de la orientación hacia el éxito económico y social y hacia los valores dominantes en la sociedad capitalista. (Jerez Mir, 1990, pp. 391-393)

Si bien el credencialismo ya se separa del funcionalismo en cuanto que deja de lado el optimismo exagerado en el poder de la educación y realiza una seria denuncia contra el sistema educativo norteamericano, la ruptura será definitiva con la llegada de la Nueva Sociología Británica de la Educación.

En efecto, al ser dejada en segundo plano la Pedagogía, como ciencia que brinda unidad a los estudios educativos, "la ciencia -o ciencias- de la educación, reducida a sociología de la educación (y en algunos casos, reducida a psicología de la educación) [...] pasará del enfoque positivista al hermenéutico y luego al crítico, que parece ser el dominante en la actualidad, aunque cuestionado" por los enfoques posmoderno y analítico, hoy todavía vigente (Vázquez, 2001, p. 15).

\subsection{Introducción de las teorías críticas}

Así, hacia la década de los setenta, se introducen críticas a aquellas teorías que, bajo criterios de racionalidad y con apariencia de objetividad y neutralidad, excluyen de sus planteos los contextos reales en los que se presentan los problemas educativos.

Como ya se anticipó, el quiebre definitivo se produce con la irrupción de la New Sociology of Education en la Universidad de Londres. En 1970 se realiza la Conferencia Anual de la British Sociological Association, cuyos temas centrales son presentados por Young, Bernstein y Bourdieu en su obra Conocimiento y control. Las bases en las que se apoya la New Sociology of Education son: la pretensión de fusionar la Sociología de la Educación con la Fenomenología y la Etnometodología; la concepción de la realidad y del conocimiento como construcciones sociales; el tratamiento sociológico del conocimiento escolar y del currículum acentuando las relaciones entre conocimiento y poder; el objetivo de desvelar la vida cotidiana en la práctica escolar, el currículum y la interacción en clase; la 
concepción de la investigación educativa como investigación en el aula; la concepción del profesor como investigador que elabora autónomamente sus propios problemas científicos y reconstruye críticamente los datos (Vázquez, 2001, p. 16).

Esta corriente surge como reacción al funcionalismo, modelo que centra su análisis en el nivel macro-sociológico de la relación entre sistema escolar y sistema social. Por contraposición, la Nueva Sociología de la Educación focaliza sus estudios en los microprocesos que se dan en la escuela y en el aula; enfatiza las representaciones de los docentes y el conocimiento escolar o currículum como forma de legitimación del poder.

En este marco, se distingue el currículum como hecho y el currículum como práctica. El primero consiste en una determinación a priori de los contenidos correspondientes a las diversas asignaturas. En el segundo caso, consiste en una construcción fruto de la interacción en el aula. Como afirma Jerez Mir:

El currículum coincide siempre con una práctica social concreta, relativamente compleja y que resulta finalmente de la interacción dialéctica objetiva entre las múltiples actividades sociales que los profesores y alumnos desarrollan en el contexto del aula y del conjunto del centro de enseñanza. Es decir, en lugar de resaltar la dimensión objetiva y acabada del conocimiento [...] esta sociología tiende a cargar el acento científico en su dimensión intersubjetiva, activa y abierta: son los hombres quienes producen y reproducen constantemente [...] el conocimiento; y son, por tanto, los profesores y los alumnos los que crean y recrean continuamente la realidad social concreta del sistema de enseñanza en cada momento histórico. (1990, p. 380)

Esta construcción y organización del conocimiento escolar (currículum) se corresponde con la distribución social del poder, lo que asegura el control social. Por esta razón, se propone una desconstrucción de saberes: se busca analizar cómo se ha ido construyendo, a partir de las diferentes interacciones, el conocimiento escolar.

Esta perspectiva fue desarrollada principalmente por Basil Bernstein, quien denuncia una correlación entre clase social, código sociolingüístico y código educativo. Plantea que las diferentes subculturas manejan diferentes códigos lingüísticos o modos de hablar y, por ende, diversos modos de razonar. Así, en tanto que la cultura de la clase alta es abstracta y universalista, la de la clase obrera sería principalmente concreta y particularista. Estos tipos de culturas se corresponden respectivamente con lo que Bernstein denomina código elaborado y código restringido. Como el lenguaje y la cultura escolar o académica son esencialmente universalistas y elaborados, los alumnos provenientes de la clase obrera se 
topan con una gran barrera de acceso a dichos códigos, lo que ocasiona graves dificultades de aprendizaje. En palabras de Bernstein "la escuela no toma en consideración la experiencia del niño, lo que hace que el niño no tome en consideración la experiencia de la escuela" (en Jerez Mir, 1990, p. 417).

Otro aporte importante es el realizado por Pierre Bourdieu, que se encuentra sintetizado en la obra La reproducción. Elementos para una teoría del sistema de enseñanza en 1970, escrita en colaboración con Passeron. Estos autores hablan sobre la teoría de la violencia simbólica y la función política del sistema de enseñanza. El concepto de violencia simbólica alude a una fuerza o poder que impone sus significaciones como legítimas y disimulando estas propias relaciones de fuerza, haciéndolas aparecer como naturales y justas, a fin de justificar la desigual situación social.

A partir del análisis de esta violencia simbólica como forma de poder estratificador, reproductor y legitimador los autores concluyen que el sistema de enseñanza moderno cumple tres funciones: una función cultural que consiste en transmitir y conservar sutilmente la cultura dominante como la cultura; una función social que reproduce la desigual estructura social y las relaciones que la mantienen y una función ideológica capaz de ocultar las otras dos funciones bajo apariencia de neutralidad y lograr así la integración intelectual y moral de los individuos. La diferenciación de estas funciones que ejerce el sistema educativo moderno y la definición del concepto de violencia simbólica han sido fundamentales para los análisis críticos y reproductivistas del siglo XX provenientes de la investigación educativa y social.

Según Stella Maris Vázquez (1994, pp. 49-50), las fuentes teóricas de la Nueva Sociología de la Educación son el interaccionismo simbólico de George Mead y la fenomenología social de Alfred Schutz. La primera de estas fuentes concibe a la sociedad como un escenario donde el individuo es un actor en interacción con otros, que negocia significados y de ese modo va construyendo su propia personalidad y la vida social. De esta perspectiva, la Nueva Sociología adopta el concepto del individuo como actor social que construye significados mediante la interacción y la metodología descriptiva de la vida de los grupos, principalmente, la observación directa. De la segunda fuente toma el concepto de la realidad social como una construcción subjetiva que debe ser descripta e interpretada. Con este punto de vista interpretativista, la Nueva Sociología pretende distanciarse del enfoque positivista que caracterizaba al funcionalismo. Correspondiéndose con estas ideas de base, la metodología de investigación utilizada consiste, especialmente, en la observación participante, entrevistas semiestructuradas y estudios de casos. 
Lo dicho caracteriza a la New Sociology of Education en su primera fase. Posteriormente, hacia fines de 1970, se introduce el neomarxismo y da lugar a una segunda fase dentro de este movimiento. Las críticas que se realizan a la primera etapa se centran en la acentuación de los micro-procesos y de la noción de la construcción social de la realidad. La influencia marxista lleva a replantear la relación entre currículum, cambio social y macrocontexto social. Por un lado, se afirma que los intereses de las clases sociales dominantes son los que dan forma a los valores que rigen la escuela, convirtiéndose esta así en factor de reproducción de la estructura social. Por otro, se comienza a tomar en cuenta las posibilidades que poseen las personas para resistir la dominación. Se introducen nociones importantes como cambio social y curricular, clase social, acción política transformadora.

Representantes destacados de esta segunda fase son Young (cuyo pensamiento fue evolucionando), Whitty, Baudelot, Establet, Bowles y Gintis. Los aportes de estos teóricos comienzan a difundirse en América Latina alrededor de los años 80.

Con este cambio en la dirección de los estudios, la misma Nueva Sociología de la Educación "engendra el paradigma que intenta ser su superación" (Vázquez, 1994, p. 53). El análisis sociológico neomarxista de las macro-estructuras dio lugar al comienzo de la pedagogía crítica. En ella también se diferencian dos etapas: la primera puede ser caracterizada como pedagogía de la reproducción y la segunda suele denominarse pedagogía de la resistencia.

Los autores reproductivistas más destacados son Althusser, Bowles y Gintis. Althusser plantea el problema de los aparatos ideológicos del Estado que contribuyen a "la reproducción de las condiciones sociales de la producción capitalista" (Jerez Mir, 1990, p.424) y al sometimiento frente a la ideología dominante. Dentro de estos aparatos se encuentran la escuela, la familia, las iglesias, los medios de comunicación, el sistema político. La escuela es el aparato ideológico del Estado que se encarga de la capacitación y diferenciación profesional de los ciudadanos. Asimismo, tiene la función ideológica de reproducir las relaciones sociales de sumisión y explotación, pero aparentando independencia y neutralidad.

Por su parte, Bowles y Gintis hablan sobre la correspondencia entre educación y empleo. Sostienen que la escuela es un instrumento de reproducción de las relaciones de dominación y subordinación sociales que se dan en función de los intereses económicos del capitalismo. De este modo, la función de la escuela consiste en perpetuar la ideología 
dominante y las desigualdades sociales y en distribuir las habilidades necesarias para la reproducción social del trabajo.

Estos autores explican cómo se da la correspondencia entre las relaciones sociales escolares (materiales y simbólicas) y las relaciones sociales del trabajo y la producción. El sistema educativo realiza una réplica del sistema productivo: acostumbra a los estudiantes a la disciplina en el trabajo; transmite formas de expresión, de comportamiento y de presentación personal adecuadas al puesto de trabajo; legitima una estructura jerárquica verticalista. Todo esto ayuda a los jóvenes a insertarse en el mundo laboral de modo no conflictivo.

El trabajo enajenado se refleja en la falta de control que tiene el estudiante sobre su educación, la enajenación de éste sobre el contenido de sus planes de estudio, y la motivación del trabajo escolar a través del sistema de calificaciones y otras recompensas externas [...]. La fragmentación del trabajo se refleja en la competencia institucionalizada y muchas veces destructiva entre estudiantes, mediante una constante evaluación y clasificación ostensiblemente meritocráticas. (Bowles y Gintis, en Vázquez, 1994, p. 433)

A su vez, los diferentes niveles escolares forman a los futuros trabajadores para ocupar distintos puestos en la estructura ocupacional. En los niveles inferiores de educación se forma para respetar reglas impuestas; en los niveles medios se educa para ser capaz de operar sin una supervisión directa y constante, pero con objetos fijados de antemano; en los niveles superiores se fomenta la libertad y autonomía y la interiorización en las normas.

Finalmente, los mencionados autores denuncian el curriculum oculto, en oposición al manifiesto. La noción de curriculum ya no se refiere tanto a una construcción social, sino a un instrumento ideológico.

Paulatinamente, esta línea de pedagogía crítica reproductivista fue dando lugar a los pedagogos de la resistencia. Como su nombre lo indica, los teóricos de esta corriente acusan a los reproductivistas la sobredimensión que realizan de la idea de dominación y la asunción de un determinismo carente de planteos que tengan en cuenta la posibilidad de una acción respecto de las estructuras económicas y de clase. Los autores más destacados son Henry Giroux, Peter Mc Laren, Paul Willis, Michael Apple.

En este enfoque se manejan conceptos clave como contradicción, conflicto, lucha, crítica, resistencia, autonomía. Las subculturas dentro de una sociedad son ámbitos de 
reproducción, pero también de resistencia. De este modo, la escuela no solo reproduce las relaciones sociales de dominación, sino que constituye también un ámbito con cierta independencia que permite resistir y contraponerse a las macroestructuras. La escuela, más que un mecanismo de reproducción ideológica, es un medio de producción de ideología y cultura a través de luchas y resistencias de clases.

El fundamento teórico de esta postura es la teoría crítica de Habermas, haciendo especial énfasis en la necesidad de asumir una práctica emancipatoria. La teoría de la resistencia concibe a la educación como radical y esencialmente política, cuyo objetivo es formar una mentalidad crítica de las instituciones y valores sociales y generar actores para la transformación social.

En cuanto a las metodologías de análisis utilizadas y a las temáticas abordadas se encuentran las historias personales o narrativas, la desconstrucción del currículum como texto donde se explicitan contradicciones que hacen al currículum oculto, la toma de autoconciencia manifestada en la expresión de la propia voz, la autoconciencia del docente y de su praxis como punto de partida para el cambio.

Son esenciales a la pedagogía crítica los conceptos de crítica y de teoría y praxis. En efecto,

[...] Mc Laren sostiene que la pedagogía crítica, como alternativa al modelo pedagógico neo-conservador de la eficiencia, une la lucha por el conocimiento crítico a la lucha por la democracia. La crítica debe ejercerse en el plano político y en el cultural, siendo este último el sitio de la disyuntura, ruptura y contradicción por lo cual la tarea crítica une teoría y praxis: la praxis es la transformación de la experiencia social y dicha transformación exige la crítica. Por ello todo conocimiento es contextual: es la conciencia de los elementos socio-culturales que han ido configurando las propias categorías de pensamiento y de lenguaje. En la 'práctica crítica' se unen teoría y praxis. (Vázquez, 1994, p. 58)

En la pedagogía crítica, entonces, se enfatiza la razón autocuestionadora que critica el status quo político y cultural y deviene necesariamente praxis, es decir, transformación social y político-cultural. Esta transformación permite el quiebre y el replanteo de las categorías conceptuales asumidas, lo que supone una nueva crítica teórica. De este modo, teoría crítica y praxis se implican y se suceden mutuamente. 
Finalmente, cabe agregar la mención que Lauder, Brown y Halsey hacen sobre la influencia actual de la Sociología de la Educación al nivel de las decisiones políticas. En efecto, argumentan que en la actualidad la influencia intelectual y política de esta disciplina ha declinado. Una de las causas es que "disciplinas como economía y política social han asumido cuestiones clave que una vez fueron la custodia de la sociología de la educación" (2009, p. 569). Por tanto, la Sociología de la Educación, según los autores, debería replantearse sus temáticas, métodos y, principalmente su "audiencia", a fin de que sus resultados influyan realmente en decisiones de política educativa.

\section{Algunos elementos que enriquecen el análisis epistemológico}

Lauder et al., realizan un análisis de la historia y la prospectiva de la Sociología de la Educación. En él postulan que las preguntas, métodos y teorías de una disciplina no son suficientes para definirla. Justifican esta afirmación aludiendo que las teorías que abarca la Sociología de la Educación son muchas y variadas y, como han ido surgiendo al reconocer diferentes y nuevos fenómenos en la escuela y la sociedad, es difícil determinar la base sociológica común que poseen. Preguntas, métodos y teorías no siempre respetan los límites de una disciplina (2009, pp. 570-571).

En consecuencia, los autores postulan que "una de las formas de entender la sociología de la educación como una disciplina es en términos institucionales, que suponen juicios acerca de las preguntas, teorías y métodos que en cualquier momento 'cuentan' como parte de la disciplina" (Lauder et al., 2009, p. 570). Lo institucional como definición de la disciplina, continúan, no implica una degradación epistemológica de la misma. Por el contrario, juega un rol importante, ya que provee una forma de iniciación, entrenamiento y trabajo para los jóvenes académicos y brinda una comunidad crítica que juzga las preguntas y teorías más significativas y la calidad del trabajo realizado.

En este marco de definición institucional de la disciplina los autores mencionados se remiten al término infraestructura de la teoría social. Esta hace referencia a los sentimientos y supuestos de la disciplina, así como a las concepciones de la realidad que forman un fundamento que influye en la elección teórica y su desarrollo.

Esta infraestructura de sentimientos y supuestos, en el caso de la sociología de la educación, se ha asociado históricamente a lo que los autores denominan "una visión

\footnotetext{
${ }^{4}$ Las traducciones realizadas a este artículo de Lauder, Brown y Halsey son nuestras en todos los casos.
} 
redentora de la educación que busca desterrar la desigualdad y permitir el desarrollo individual" (Lauder et al., 2009, p. 571).

Por su lado, el examen del origen de la Sociología de la Educación como disciplina permite observar que la intención con la que surge no es complementar los estudios pedagógicos que se venían realizando, sino redireccionarlos. En sus inicios durkheimianos esta disciplina no pretende complementar la tradición alemana de Pedagogía, sino plantear un nuevo tratamiento de los problemas educativos.

Tanto en el primer momento positivista como en la transición hacia enfoques críticos, la finalidad de la Sociología de la Educación es estudiar la educación como un fenómeno eminentemente social. Esto significa concebir la educación como un fenómeno que se lleva a cabo y se define en una sociedad y por una sociedad: los fines de la educación no son establecidos según los fines del hombre, sino según lo que la sociedad determina que es necesario inculcar en el educando; los contenidos del acto educativo son definidos según las necesidades y exigencias que la sociedad manifiesta. Un ejemplo actual, en el cual los fines y medios educativos, así como la concepción de hombre que se desea educar están definidos desde un abordaje eminentemente sociológico, es el enfoque de la educación por competencias o para el trabajo. Dice al respecto Muñoz Varela:

Se aboga por una educación basada en el desarrollo de habilidades y destrezas que permita dotar a las personas de una formación acorde con los requerimientos de competitividad, innovación, eficiencia, eficacia y flexibilidad, los cuales, en el marco de la globalización económica y de los intensos desarrollos de la SIC [Sociedad de la Información y Comunicación], hoy se instituyen como principios privilegiados con base en los cuales han de organizarse y llevarse a cabo en general todas las actividades humanas. (2012, pp. 27-28)

Es decir, como se observa en su origen y evolución, la Sociología de la Educación no procura estudiar una dimensión más de la persona, la sociabilidad, en un intento de complementar los estudios que puedan llevar a cabo otras disciplinas. Procura realizar el único abordaje que supone puede hacerse de la educación como objeto. Esto es, el estudio de la educación como un fenómeno exclusivamente social, cuyos principios, fines y contenidos, así como las políticas y las instituciones en las que tiene lugar, son definidos por y según la sociedad en la que se desarrolla. Y será justamente este tipo de abordaje el que 
permitirá inferir, desde este enfoque sociológico, principios y pautas para consolidar también la personalidad del estudiante y el docente.

La Sociología de la Educación, en cuanto que es una de las llamadas Ciencias de la Educación ${ }^{5}$, tiene como objeto compartido por todas ellas la educación. Sin embargo, puede observarse a partir del examen de su evolución histórica que la Sociología de la Educación define para sí misma una noción específica de educación, que no es necesariamente compartida por las restantes ciencias educativas.

El problema que comienza a advertirse es que ese objeto común a las ciencias de la educación no es concebido de igual modo por todas estas ciencias. $Y$ el asunto es que las diferencias entre estas concepciones no se refieren solamente a la porción de ese objeto común a la que cada ciencia se dirige, o al modo (método) de acercarse a él. Las diferencias son más bien sustanciales, cambia en esencia entre una y otra disciplina la noción de educación y de ciencia.

Tenti Fanfani reafirma esta constatación:

Este campo intelectual, a diferencia de otros campos científicos, se caracteriza por la existencia de productores procedentes de disciplinas muy diversas. El pluralismo disciplinario es una característica distintiva de este campo que por una parte es una ventaja y por la otra la fuente de una serie de obstáculos. En efecto, son muchos los lenguajes con que se habla de 'las cosas de la educación'. [...] Esta variedad es una riqueza, pues la diversidad de lenguajes permite ver más dimensiones y matices del hecho educativo. En verdad, podríamos decir que lo que unifica a los investigadores educativos' no es un objeto teórico, sino un objeto empírico común: las instituciones (reglas y recursos), agentes, procesos y productos educativos. No es raro que esta ausencia de un lenguaje común dificulte el diálogo y el control recíproco entre los productores. (2010, p. 63)

A esta situación se le suma lo que plantean Lauder, Brown y Halsey sobre la definición de la Sociología de la Educación no desde sus preguntas, métodos y teorías, sino desde lo institucional.

En efecto, esta disciplina, así como varias de las ciencias de la educación, surgió a partir de teorías o enfoques que concebían de un modo particular el objeto educación. Con el

\footnotetext{
${ }^{5}$ Se entiende por ciencias de la educación el conjunto de disciplinas científicas que estudian la educación en alguna de sus facetas o alguno de sus elementos. Así, se habla de Teoría de la Educación, Didáctica, Filosofía de la Educación, Psicología de la Educación, Sociología de la Educación, Política Educativa, entre otras.
} 
paso del tiempo estas teorías se institucionalizaron como disciplina científica: tuvieron gran difusión en los ámbitos académicos, se escribieron libros y artículos al respecto, se editaron revistas, se conformaron carreras universitarias y se emitieron certificados y habilitaciones profesionales en relación a ella. Estos factores institucionales influyeron decisivamente para que esas teorías y otras se configuraran como Ciencias de la Educación.

En efecto, los planes de estudio universitarios de formación de maestros y profesores contemplan una serie de asignaturas, que se corresponden con alguna de las ciencias de la educación (por ejemplo, Filosofía de la Educación, Didáctica, Sociología de la Educación, Teoría Curricular, Psicología de la Educación, entre otras) y que se enseñan como complementarias. Lo que se supone aquí es que el futuro docente incorporaría una serie de nociones provenientes de estas ciencias educativas que, en su reunión, proporcionarían un conocimiento y unas habilidades prácticas que lo ayudarían en su tarea docente.

Esta suposición queda cuestionada si se advierte lo observado anteriormente: el objeto educación no solo se aborda desde diferentes ángulos, según las distintas disciplinas, sino que se concibe de diferente modo en cada una de ellas.

De este modo queda planteado el problema de la inconmensurabilidad. Con este término se enuncia la tesis según la cual dos teorías científicas resultan incomparables, dado que no existe un patrón o criterio común extrínseco a ellas a partir del cual estas puedan compararse, juzgarse y posicionarse una como mejor que la otra. En la década de 1960 coincidieron Thomas Kuhn y Paul Feyerabend en la postulación de esta tesis: "las grandes teorías científicas son inconmensurables entre sí, y ni el recurso a experimentos ni la argumentación lógica son criterios válidos que permitan una justa comparación entre ellas. [...] Teorías distintas definen diversos hechos, provocan diversos experimentos y, por tanto, interpretan de diverso modo los supuestos resultados objetivos" (Gelonch Villarino, 2010).

En consecuencia, si cada ciencia de la educación define ella misma para sí qué es educación, enseñanza, aprendizaje, conocimiento, curriculum, etc., estos conceptos resultan muy diferentes, y a veces opuestos, en una y otra disciplina. Por lo tanto, se hace difícil obtener acuerdos interdisciplinares en relación a qué es y cómo debe ser educar. Por consiguiente, también se dificulta la aplicación práctica de los principios o resultados obtenidos en cada una de las Ciencias de la Educación. En efecto, el docente puede percibir que estos resultados no siempre pueden orientarlo de manera complementaria en la toma de decisiones a nivel áulico, institucional o político. Y esto se entiende si se comprende que cuando las diferentes ciencias hablan de educador, educando, conocimiento, no se están 
refiriendo a lo mismo en todos los casos. Inmediatamente salta a la vista el problema: el futuro profesor recibe herramientas provenientes de las diversas ciencias educativas que no siempre son complementarias, por lo que no siempre pueden ayudarle eficazmente en su práctica. Esto se agudiza si se acepta que la realidad sobre la que tiene que actuar el docente es sumamente problemática: "los graduados universitarios enfrentan retos diariamente que les exigen integrar sus conocimientos, nuevas capacidades, y adaptar sus aprendizajes a nuevos contextos y formas de complejidad" (Thompson Klein en Alfaro, Badilla \& Miranda., 2012, p. 3).

Este no sería un problema si la educación se viera como un acto a cuyo estudio concurren distintas disciplinas procedentes de diferentes áreas del saber. El problema se presenta cuando esas disciplinas se denominan Ciencias de la Educación y presentan sus abordajes como complementarios en la comprensión de ese objeto que, evidentemente, no es concebido de igual modo por ellas.

En la formación del futuro docente estos problemas epistemológicos se hacen patentes y se fenomenizan en la práctica. Así, ante una situación áulica concreta, el profesor puede percibir que no cuenta con suficientes guías u orientaciones teóricas y prácticas para llevar adelante su clase. Se sigue viendo la buena enseñanza como una mezcla de instinto, buen criterio y algo de experiencia; pero el profesor que enseña matemática, física, música o literatura no siempre acude a los conocimientos pedagógicos recibidos en su formación docente como una fuente confiable de orientación en la práctica.

En todo caso, comenzar a sacar a la luz estas cuestiones epistemológicas subyacentes, que en este trabajo se han ejemplificado con el caso de la Sociología de la Educación, contribuye a ganar claridad en la comprensión del problema y a pensar en caminos alternativos en la formación pedagógica de profesores y maestros. Caminos que deberán intentar comprender cada ciencia educativa desde sí misma y arribar a una visión integral de la educación que pueda dar unidad al conjunto.

\section{Síntesis y reflexiones finales}

Se ha visto que la Sociología de la Educación surgió en la modernidad para estudiar la educación como un hecho social que debe ser descrito causalmente. En este sentido, la educación es el fenómeno que permite a las sociedades transmitir a las generaciones jóvenes las pautas de comportamiento que desea para mantener la necesaria homogeneidad y diversificar el trabajo para lograr una interdependencia funcional. 
Con estas características Durkheim abre el camino a la Sociología de la Educación como la ciencia de la educación. A partir de allí, la disciplina recorre distintos abordajes sociológicos que culminan con el enfoque crítico de los pedagogos de la reproducción y la resistencia.

Se vio que, tanto en los planteos positivistas como en los críticos, esta ciencia hace una aproximación a la educación como hecho social. Se sostiene, dentro de esta disciplina, que es la mirada sociológica la que puede ofrecer una visión auténtica de qué es la educación y cómo deberían ser los curricula, las instituciones y las políticas educativas.

Esta situación permite inferir que, así como la Sociología de la Educación, las distintas Ciencias de la Educación, que formarían parte del mismo campo justamente por compartir el objeto educación, definen de modo particular qué es educación y qué es ciencia. Por ello, queda en evidencia la inconmensurabilidad que subyace a los estudios educativos y que se manifiesta en la percepción de una confusión o escasa aplicabilidad práctica de los aportes pedagógicos en la formación docente.

Finalmente, quedó planteada la necesidad de seguir profundizando en las cuestiones epistemológicas que permanecen en el fondo de los estudios pedagógicos y de transitar hacia comprensiones no tan parciales de la educación y de los elementos que en ella intervienen. En otras palabras, quedó planteada la necesidad de lograr una verdadera integración de los contenidos, resultados, métodos, herramientas y teorías provenientes de las distintas Ciencias de la Educación para acercarse a la solución de problemas complejos y lograr una comprensión de lo fundamental.

\section{Referencias}

Alfaro Castegnaro, Milena, Badilla Saxe, Eleonora y Miranda Garnier, Ximena. (2012). Hacia la transdisciplinariedad en la docencia en la Universidad de Costa Rica. Actualidades Investigativas en Educación, 12(1), 1-37. Recuperado de: http://revista.inie.ucr.ac.cr/uploads/tx magazine/hacia-transdisciplinariedad-docenciauniversidad-costa-rica-alfaro-badilla-miranda.pdf

Durkheim, Emilio. (1976). Educación y sociología (Trad. Alice Pestana). Madrid: La Lectura.

Gelonch Villarino, Santiago. (2010). Voz “inconmensurabilidad”. En Ángel González (ed.), Diccionario de filosofía. Pamplona: EUNSA.

Guerrero Serón, Antonio. (1996). Manual de Sociología de la Educación. Madrid: Síntesis. Jerez Mir, Rafael. (1990). Sociología de la Educación. Madrid: Consejo de Universidades. 
Lauder, Hugh, Brown, Phillip y Halsey, A. H. (2009). Sociology of Education: a critical history and prospects for the future. Oxford Review of Education. 35(5) 569-585.

Muñoz Varela, Luis. (2012). Enfoque por competencias y mercado de trabajo: Nuevas tendencias para la educación universitaria. Actualidades Investigativas en Educación, 12 (2) 1-30. Recuperado de http://revista.inie.ucr.ac.cr/uploads/tx magazine/enfoquecompetencias-mercado-trabajo-nuevas-tendencias-educacion-universitaria-munoz.pdf

Quintana Cabanas, José María. (1989). Sociología de la educación. Madrid: Dykinson.

Rodríguez Sedano, Alfredo. (2006). Hacia una fundamentación epistemológica de la pedagogía social. Educación y educadores, 9(2), 131-147. Recuperado de http://educacionyeducadores.unisabana.edu.co/index.php/eye/article/view/667

Tenti Fanfani, Emilio. (2010). Notas sobre la estructura y dinámica del campo de las Ciencias de la Educación. Espacios en blanco. Revista de educación. Serie Indagaciones, 20, 57-79. Buenos Aires: UNCPBA.

Vázquez, Stella Maris. (1994). La teoría del curriculum en la actualidad. Buenos Aires: CIAFIC.

Vázquez, Stella Maris. (2001). La Filosofía de la Educación: Estado de la cuestión y líneas esenciales. Buenos Aires: CIAFIC. 